\title{
Redefining Tumor Burden in Patients with Intermediate-Stage Hepatocellular Carcinoma: The Seven-Eleven Criteria
}

\author{
Ya-Wen Hung ${ }^{a}$ I-Cheng Lee ${ }^{a, b}$ Chen-Ta Chi ${ }^{a, b, c}$ Rheun-Chuan Lee ${ }^{d}$ \\ Chien-An Liu $^{d}$ Nai-Chi Chiu ${ }^{d}$ Hsuen-En Hwang ${ }^{d}$ Yee Chao $^{e}$ \\ Ming-Chih Hou ${ }^{a, b}$ Yi-Hsiang Huang ${ }^{a, b, c}$
}

aDivision of Gastroenterology and Hepatology, Department of Medicine, Taipei Veterans General Hospital, Taipei, Taiwan; ${ }^{b}$ Faculty of Medicine, National Yang Ming Chiao Tung University School of Medicine, Taipei, Taiwan; Institute of Clinical Medicine, National Yang Ming Chiao Tung University, Taipei, Taiwan; 'Department of Radiology, Taipei Veterans General Hospital, Taipei, Taiwan; ${ }^{~}$ Cancer Center, Taipei Veterans General Hospital, Taipei, Taiwan

\section{Keywords}

Hepatocellular carcinoma · Transarterial

chemoembolization - Tumor burden

\begin{abstract}
Background and Aims: For patients with intermediate-stage hepatocellular carcinoma (HCC), the definition of high tumor burden remains controversial. This study aimed to compare the prognostic value of different criteria of tumor burden in patients with intermediate-stage HCC undergoing transarterial chemoembolization (TACE). Methods: From 2007 to 2019, 632 treatment-naive patients with intermediate-stage HCC undergoing TACE were retrospectively enrolled. We compared different criteria of tumor burden in discriminating radiologic response and survival, including up-to-7, upto-11, 5-7, 7 lesions criteria, and newly proposed 7-11 criteria. Results: The proportions of patients classified as high tumor burden were varied by different criteria. Among the 5 criteria, 7-11 criteria have the best performance to discrimi-
\end{abstract}

nate complete response (CR) and overall survival (OS) after TACE. In patients with low, intermediate, and high tumor burden classified by $7-11$ criteria, the CR rate was 21,12 , and $2.5 \%$, respectively ( $p<0.001$ ), and the median OS was 33.1, 22.3 , and 11.9 months, respectively $(p<0.001)$. By multivariate analysis, 7-11 criteria were significantly associated with $\mathrm{CR}$ (intermediate vs. high burden, odds ratio $=4.617, p=$ 0.002 ; low vs. high burden, odds ratio $=8.675, p<0.001$ ) and OS (intermediate vs. high burden, hazard ratio $=0.650, p<$ 0.001 ; low vs. high burden, hazard ratio $=0.520, p<0.001$ ). Seven to 11 criteria also had the lowest corrected Akaike information criteria, highest homogeneity value, and highest area under the receiver operating characteristic curve in predicting 1-, 2-, and 3-year mortality among all criteria. Conclusion: Conventional definitions of tumor burden were not optimal for patients with intermediate HCC. The new 7-11 criteria had the best discriminative power in predicting radiologic response and survival in patients with intermediate-stage HCC undergoing TACE.

(c) 2021 The Author(s).

Published by S. Karger AG, Basel
(C) 2021 The Author(s)

Published by S. Karger AG, Basel

This is an Open Access article licensed under the Creative Commons Attribution-NonCommercial-4.0 International License (CC BY-NC) (http://www.karger.com/Services/OpenAccessLicense), applicable to the online version of the article only. Usage and distribution for commercial purposes requires written permission.
Correspondence to:

I-Cheng Lee, iclee@vghtpe.gov.tw

Yi-Hsiang Huang, yhhuang@vghtpe.gov.tw 


\section{Introduction}

Hepatocellular carcinoma (HCC) is the sixth most commonly diagnosed cancer and the fourth leading cause of cancer-related death worldwide [1]. For patients with intermediate-stage HCC, transarterial chemoembolization (TACE) is the guideline-recommended standard treatment [2-4]. However, intermediate-stage HCC is composed of a very heterogeneous disease with a wide range of tumor burden and liver function status, and not all patients could benefit from TACE [5]. In particular, high tumor burden is considered an important component adopted by various subclassifications or prediction models to select patients unsuitable for TACE [6]. New treatment strategies, such as early initiation of systemic therapies, have been proposed for patients who might be unsuitable or refractory to TACE treatment [7-9].

The definition of high tumor burden varied among different subclassifications and prediction models. Currently, up-to-7 is the most commonly used criteria to define high tumor burden in intermediate-stage HCC, which were adopted by Bolondi's subclassifications [10], Kinki criteria [11], and STATE score [12]. Nevertheless, the up-to-7 criteria were originally developed for patients with early-stage HCC considering liver transplantation [13] and might not be best for stratifying tumor burden in patients with intermediate-stage HCC. Recent studies showed that up-to-11 criteria could be more discriminative than up-to-7 criteria for predicting survival after TACE $[14,15]$, whereas 5-7 criteria and 7 lesions criteria have also been proposed to stratify tumor burden of intermediate-stage HCC [6]. Therefore, the most optimal definition of high tumor burden for patients with intermediate-stage HCC remains debated. A better definition of high tumor burden may help select patients suitable for TACE and to predict survival after TACE.

The objective radiologic response, which could be achieved in about $40-50 \%$ of patients after TACE, was also an important predictor of better survival in patients treated with TACE $[16,17]$. Patients with high tumor burden may have lower response rate and higher progression rate to TACE. A combination of systemic therapies and TACE has been shown to increase the response rate and survival in these patients [18]. The optimal definition of tumor burden to predict radiologic response to TACE was also unclear. The aim of this study was to compare different criteria of tumor burden in predicting radiologic response and survival in patients with intermediatestage HCC undergoing TACE.

\section{Patients and Methods}

\section{Patients}

From January 2007 to January 2019, 1,136 treatment-naive HCC patients who were initially treated with TACE in Taipei Veterans General Hospital were retrospectively reviewed. Among them, 632 patients belonged to intermediate-stage HCC were enrolled. The diagnosis of HCC and TACE eligibility was assessed before treatment using contrast-enhanced computed tomography (CECT) or magnetic resonance imaging (MRI), which fulfilled the diagnostic criteria of the American Association for the Study of Liver Diseases (AASLD) treatment guidelines [19].

\section{TACE Procedure}

After tumor stain detection and tumor feeding artery navigation, superselective catheterization into tumor-feeding branches was performed with a $1.98-/ 2.5-\mathrm{Fr}$ microcatheter advanced through a 4-/5-Fr catheter (Terumo, Tokyo, Japan or Cook Medical, Bloomington, IN, USA) and subsegmental TACE was performed with emulsion containing 5-30 mg of doxorubicin (Actavis, Dublin, Ireland) and $0.5-15.0 \mathrm{~mL}$ of lipiodol (Laboratoire Guerbet, Paris, France), followed by the delivery of $1-\mathrm{mm}^{2} \mathrm{Gel}-$ foam cubes (SPONGOSTAN ${ }^{\mathrm{TM}}$; Ferrosan Medical Devices, Søborg, Denmark). The total amount of iodized oil was determined based on the tumor size and liver function. All target tumors were evaluated by revealing reduced antegrade tumor-feeding arterial flow and reduced or no tumor stain as the embolization endpoint according to subjective angiographic chemoembolization endpoint levels 2 and 3 [14, 20]. Cone-beam computed tomography angiographic system (Axiom-Artis Zee; Siemens) equipped with a $30 \times$ $40-\mathrm{cm}^{2}$ flat-panel detector was introduced to our hospital in 2014 [21].

Embolization to all nodules was performed whenever possible. If all nodules could be embolized in 1 session, the radiologic response was evaluated by CECT or MRI 1-2 months after first session of TACE. If the tumors were too large to be embolized at once, sequential embolization would be arranged 1 month later. In this case, the radiologic response was evaluated by CECT or MRI 1-2 months after completion of 2 sessions of TACE. In cases of viable or residual tumors, repeated TACE or other treatment modalities, such as surgery, local ablation, radiotherapy or systemic therapy, was arranged if the general condition was acceptable. If subsequent TACE or multimodality treatment was not suitable, best supportive care was given.

\section{Baseline Evaluation}

We recorded detailed demographic profile, tumor characteristics, and biochemistry data. The data included age, gender, alcohol intake, hepatitis B surface antigen, anti-hepatitis $C$ virus antibody, tumor size, tumor number, serum AFP, total bilirubin, albumin, alanine aminotransferase (ALT), aspartate aminotransferase (AST), creatinine levels, and platelet count. The performance status was evaluated by using the Eastern Cooperative Oncology Group (ECOG) performance scale. The ALBI score was calculated using the formula: linear predictor $=(\log 10$ bilirubin $\mu \mathrm{mol} / \mathrm{L} \times$ $0.66)+($ albumin $\mathrm{g} / \mathrm{L} \times-0.085)$, where bilirubin is in micromole per liter and albumin in gram per liter; and the cut points of the ALBI grade were as follows: $\mathrm{xb} \leq-2.60$ (ALBI grade 1$),>-2.60$ to $\leq-1.39$ (ALBI grade 2) and $\mathrm{xb}>-1.39$ (ALBI grade 3 ) [22]. 
Table 1. Characteristics of 632 patients with intermediate-stage HCC undergoing TACE

\begin{tabular}{|c|c|}
\hline & All patients $(n=632)$ \\
\hline Age, years, mean $\pm S D$ & $69.4 \pm 12.4$ \\
\hline Gender, male, $n(\%)$ & $489(77.4)$ \\
\hline Alcoholism, $n(\%)$ & $85(13.7)$ \\
\hline HBsAg-positive, $n(\%)$ & $278(45.5)$ \\
\hline Anti-HCV-positive, $n(\%)$ & $208(34.2)$ \\
\hline ECOG status $0 / 1 / 2, n(\%)$ & $592 / 23 / 6(95.3 / 3.7 / 1.0)$ \\
\hline Child-Pugh class A/B, $n(\%)$ & $502 / 130(79.4 / 20.6)$ \\
\hline ALBI grade $1 / 2 / 3, n(\%)$ & $181 / 384 / 45(29.7 / 63.0 / 7.4)$ \\
\hline Bilirubin, $\mathrm{mg} / \mathrm{dL}$, mean $\pm \mathrm{SD}$ & $1.00 \pm 0.75$ \\
\hline Albumin, $\mathrm{g} / \mathrm{dL}$, mean $\pm \mathrm{SD}$ & $3.57 \pm 0.55$ \\
\hline $\mathrm{ALT}, \mathrm{U} / \mathrm{L}$, mean $\pm \mathrm{SD}$ & $61.3 \pm 50.1$ \\
\hline $\mathrm{AST}, \mathrm{U} / \mathrm{L}$, mean $\pm \mathrm{SD}$ & $78.7 \pm 89.0$ \\
\hline Creatinine, $\mathrm{mg} / \mathrm{dL}$, mean $\pm \mathrm{SD}$ & $1.17 \pm 1.11$ \\
\hline Platelet, $10^{9} / \mathrm{L}$, mean $\pm \mathrm{SD}$ & $159 \pm 85$ \\
\hline Tumor size, $\mathrm{cm}$, mean $\pm \mathrm{SD}$ & $6.79 \pm 3.89$ \\
\hline AFP, ng/mL, median (IQR) & $50.94(7.93-736.86)$ \\
\hline Use of cone-beam computed tomography, $n(\%)$ & $209(33.1)$ \\
\hline \multicolumn{2}{|l|}{ Tumor nodules, $n(\%)$} \\
\hline $1 / 2-3 / 4-7 />7$ & $220(34.8) / 188(29.7) / 91(14.4) / 133(21)$ \\
\hline Up-to-7 criteria, in/out & $185 / 447(29.3 / 70.7)$ \\
\hline Up-to-11 criteria, in/out & $409 / 223(64.7 / 35.3)$ \\
\hline $5-7$ criteria, in/out & $465 / 167(73.6 / 26.4)$ \\
\hline 7 lesions criteria, in/out & $499 / 133(79 / 21)$ \\
\hline 7-11 criteria, low/intermediate/high & $185 / 224 / 223(29.3 / 35.4 / 35.3)$ \\
\hline \multicolumn{2}{|l|}{ Radiologic response, $n(\%)$} \\
\hline Complete response & $67(11.5)$ \\
\hline PR & $167(28.5)$ \\
\hline SD & $168(28.7)$ \\
\hline $\mathrm{PD}$ & $183(31.3)$ \\
\hline
\end{tabular}

HCC, hepatocellular carcinoma; TACE, transarterial chemoembolization; HBsAg, hepatitis B surface antigen; ALT, alanine aminotransferase; AST, aspartate aminotransferase; ECOG, Eastern Cooperative Oncology Group; $\mathrm{PD}$, progressive disease; $\mathrm{PR}$, partial response; IQR, interquartile range; $\mathrm{SD}$, stable disease.

We compared different tumor burden criteria, including upto-7, up-to-11, 7 lesions, and 5-7 criteria, in predicting radiologic response and survival (see online suppl. Table 1; for all online suppl. material, see www.karger.com/doi/10.1159/000517393). The up-to-7 criteria combined the number of tumors and size of the largest tumor, with the sum being no more than 7 [13], whereas the up-to-11 criteria combined the number of tumors and size of the largest tumor, with the sum being no more than 11 [15]. Seven lesions criteria defined high tumor burden as total tumor numbers $>7$, while 5-7 criteria defined high tumor burden as total tumor numbers $>7$ or tumor nodules $4-7$ with the largest tumor $>5 \mathrm{~cm}$ [6]. Our results showed that patients within up-to-7 criteria had the highest chance of complete response (CR), whereas patients beyond up-to-11 criteria had the lowest chance of CR and highest chance of progressive disease (PD). Therefore, we proposed new 7-11 criteria combining the number of tumors and size of the largest tumor, which defined high tumor burden as the sum $>11$, intermediate tumor burden as the sum $>7$ but no more than 11 , and low tumor burden as the sum no more than 7 .

Redefining Tumor Burden of Intermediate HCC

\section{Outcome Assessment}

Radiologic response was evaluated according to the modified Response Evaluation Criteria in Solid Tumors (mRECIST) criteria: $\mathrm{CR}$ - the disappearance of any intra-tumoral arterial enhancement in all target lesions; partial response (PR) - at least a 30\% decrease in the sum of the diameters of viable target lesions; PD - an increase of at least $20 \%$ in the sum of the diameters of viable target lesions; and stable disease (SD) - any cases that do not qualify for either PR or PD [23]. Objective response rate (ORR) was defined as the percentage of patients with a CR or PR. The disease control rate (DCR) was defined as the percentage of patients with $\mathrm{CR}, \mathrm{PR}$, or $\mathrm{SD}$. In patients achieving CR, recurrence-free survival (RFS) was defined as the time interval between the day of the first session of TACE and HCC recurrence. Overall survival (OS) was defined as the time interval between the day of the first session of TACE and death.

\section{Statistical Analysis}

Values were expressed as median (interquartile range) or as mean \pm SD when appropriate. Pearson $\chi^{2}$ analysis was used to 
compare categorical variables. The Mann-Whitney U test was used to compare continuous variables. The Kaplan-Meier method was used to estimate survival rates. The log-rank test was used to compare survival curves between patient groups. Analysis of prognostic factors for survival was performed using the Cox proportional hazards model. Variables that achieved statistical significance $(p<$ $0.05)$ or those close to significance $(p<0.1)$ by univariate analysis were subsequently included in the multivariate analysis. Comparison of prognostic performance of different criteria of high tumor burden was calculated by corrected Akaike information criteria (AICc) and homogeneity. We used area under the receiver operating characteristic curve (AUROC) for binary class and multi-class to test each criterion's diagnostic ability in predicting survival [24]. Statistical significance was determined by 2 -tailed tests and considered statistically significant when a $p$ value $<0.05$. All statistical analyses were conducted using IBM SPSS Statistics for Windows, version 26 (IBM, Armonk, NY, USA), and R software, version 3.6.1 (R Foundation for Statistical Computing, Vienna, Austria).

\section{Results}

\section{Patient Characteristics}

The characteristics 632 patients with intermediatestage HCC initially treated with TACE were shown in Table 1 . The mean age was 69.4 years, and the patients were male-predominant (77.4\%). Most of the HCC etiologies were hepatitis B virus (44.5\%) and hepatitis C virus (34.2\%) infection. The majority of patients belonged to Child-Pugh class A (79.4\%) and ALBI grade 2 (63\%). By up-to-7, up-to-11, 5-7, and 7 lesions criteria, 70.7, 35.3, 26.4 , and $21 \%$ of patients were defined as high tumor burden, respectively. By 7-11 criteria, 29.3, 35.4, and 35.3\% of patients were defined as low, intermediate, and high tumor burden, respectively.

\section{Distributions of Radiologic Response by Different Criteria of Tumor Burden}

The median follow-up period was 19.9 months. Among the 632 patients, $11.5,28.5,28.7$, and $31.3 \%$ of patients had CR, PR, SD, and PD after the first session of TACE, respectively (Table 1). Figure 1 showed the distributions of radiologic response by different criteria of tumor burden. The rates of CR, ORR, and DCR in patients with high and low tumor burden by up-to-7 criteria were 7.3, 35.9, and $66.7 \%$, and $21,49.4$, and $73.3 \%$, respectively (Fig. 1a, $p<0.001)$. The corresponding response rates in patients high and low tumor burden were $2.5,23.5$, and $59.5 \%$, and $16.1,48.6$, and $73.5 \%$, respectively, by up-to- 11 criteria (Fig. $1 \mathrm{~b}, p<0.001$ ); 5.8, 29.7, and 60.7\%, and 13.5, 43.7, and $71.6 \%$, respectively, by $5-7$ criteria (Fig. $1 c, p=0.007$ ); $6.5,30.7$, and $59.7 \%$, and $12.8,42.5$, and $71.1 \%$, respectively, by 7 lesions criteria (Fig. $1 \mathrm{~d}, p=0.035$ ). By $7-11$ criteria, the rates of CR, ORR, and DCR were 2.5, 23.5, and $59.5 \%$, respectively; in patients with high tumor burden, $12,47.9$, and $73.7 \%$, respectively; in patients with intermediate tumor burden; and $16.1,48.6$, and $73.5 \%$, respectively, in patients with low tumor burden (Fig. 1e, $p<0.001)$.

\section{Factors Associated with Radiologic Response}

We further analyzed the predictors of radiologic response, including CR, objective response and PD after the first session of TACE. In univariate analysis, age, hepatitis B surface antigen, AFP, ALT, AST levels, 5-7 criteria, 7 lesions criteria, and 7-11 criteria were significantly associated with CR after TACE. In multivariate analysis, AFP $>200 \mathrm{ng} / \mathrm{mL}$ (odds ratio $[\mathrm{OR}]=0.432, p=0.017$ ) and $7-11$ criteria (intermediate vs. high tumor burden, $\mathrm{OR}=4.617$, $p=0.002$; low vs. high tumor burden, $\mathrm{OR}=8.675, p<$ 0.001 ) were independent predictors of $C R$ after first TACE session (Table 2).

Regarding objective response, age, AFP, AST levels, platelet count, ALBI grade 2-3, 5-7 criteria, 7 lesions criteria, and 7-11 criteria were significant factors in univariate analysis. In multivariate analysis, ALBI grade 2-3 $(\mathrm{OR}=0.613, p=0.009), 7-11$ criteria (intermediate vs. high tumor burden, $\mathrm{OR}=3.108, p<0.001$; low vs. high tumor burden, $\mathrm{OR}=3.290, p<0.001$ ) were independent predictors of objective response after TACE (online suppl. Table 2).

For tumor progression, high tumor burden defined by 5-7 criteria, 7 lesions criteria, and 7-11 criteria showed significant association with PD in univariate analysis. In multivariate analysis, 7-11 criteria (intermediate vs. high tumor burden, $\mathrm{OR}=0.525, p=0.002$; low vs. high tumor burden, $\mathrm{OR}=0.535, p=0.005)$ were the independent predictor of PD after TACE (online suppl. Table 3).

\section{Factors Associated with RFS after Achieving Complete} Response

Of the 67 patients achieving CR after the first TACE, 54 patients $(80.6 \%)$ encountered HCC recurrence during the follow-up period. The median RFS was 8.2 months (interquartile range 4.97-18.55 months). By multivariate analysis, ALBI grade 2-3 (hazard ratio $[\mathrm{HR}]=1.969, p=$ $0.025), 7-11$ criteria (intermediate vs. high tumor burden, $\mathrm{HR}=0.168, p=0.003$; low vs. high tumor burden, $\mathrm{HR}=$ $0.087, p<0.001)$ were significantly associated with RFS (online suppl. Table 4). The median RFS of patients with low, intermediate, and high tumor burden by $7-11$ criteria were $15.8,8.2$, and 4.9 months, respectively $(p<0.001$, Fig. 2a). Of note, all patients classified to high tumor bur- 

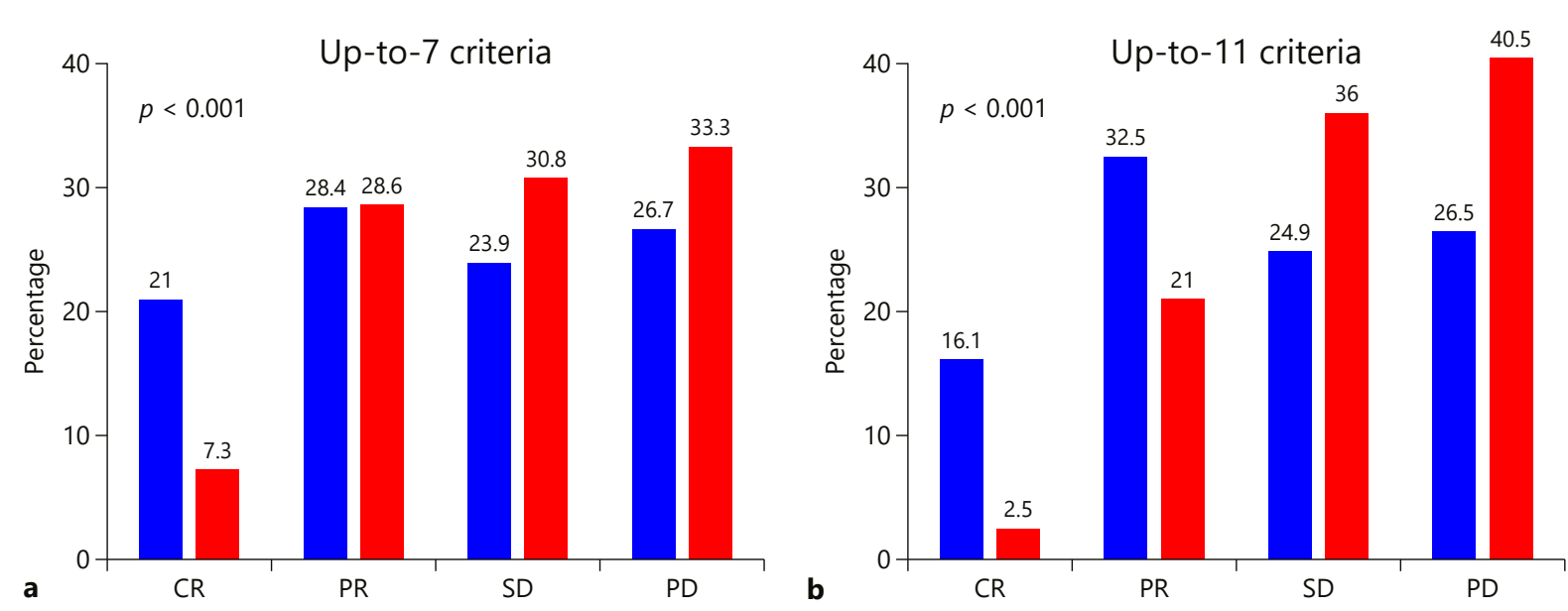

5-7 criteria
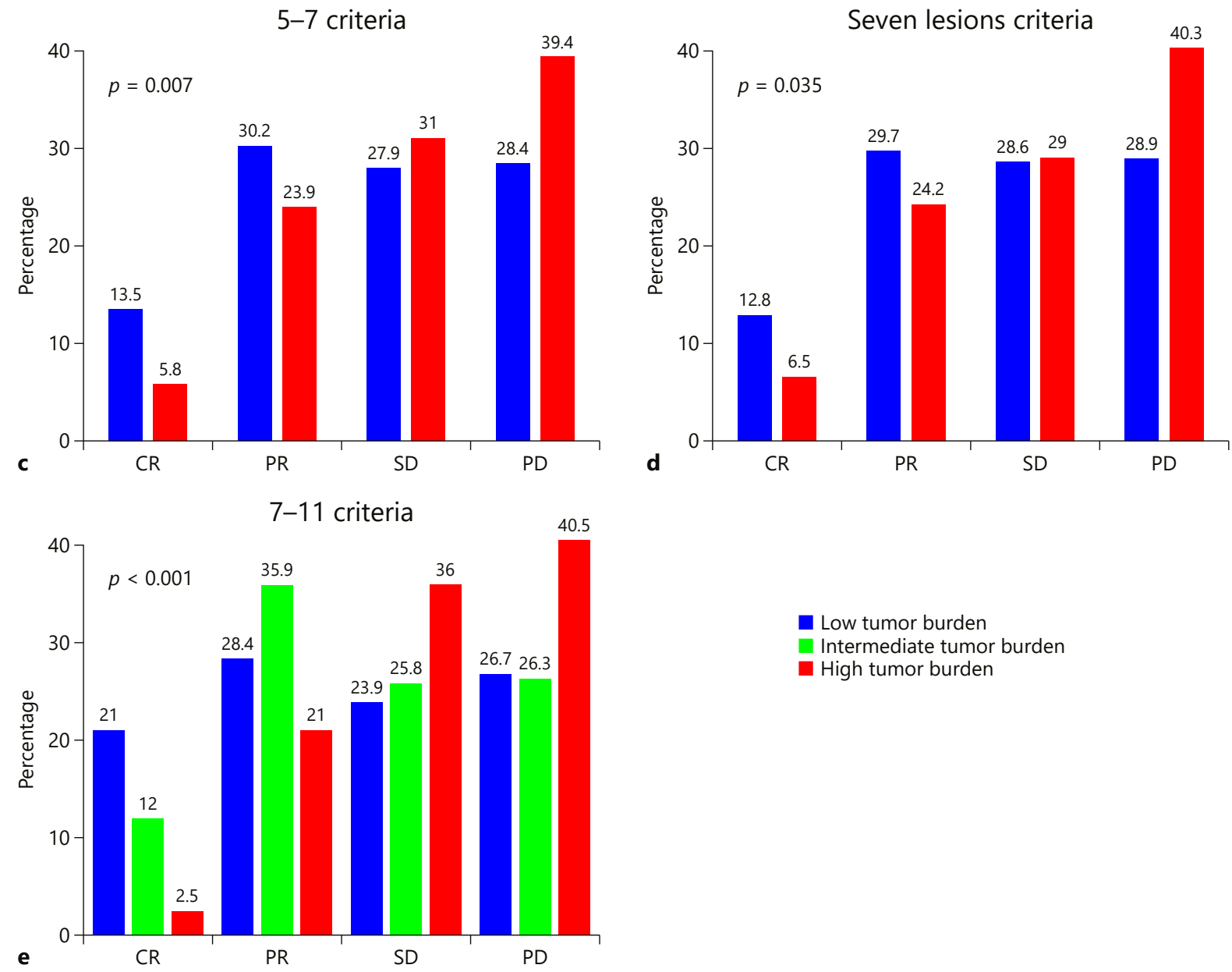

Fig. 1. Comparison of different criteria of high tumor burden and distribution of radiologic response after TACE. a Up-to-7 criteria. b Up-to-11 criteria. c 5-7 criteria. d Seven lesions criteria. e 7-11 criteria. CR, complete response; $\mathrm{PR}$, partial response; SD, stable disease; $\mathrm{PD}$, progressive disease; TACE, transarterial chemoembolization. 
Table 2. Univariate and multivariate analyses of factors associated with complete response after TACE

\begin{tabular}{|c|c|c|c|c|}
\hline & \multicolumn{2}{|l|}{ Univariate } & \multicolumn{2}{|l|}{ Multivariate } \\
\hline & OR (95\% CI) & $p$ value & OR (95\% CI) & $p$ value \\
\hline \multicolumn{5}{|l|}{ Age, years } \\
\hline$>60 / \leq 60$ & $2.364(1.141-4.898)$ & 0.021 & & NS \\
\hline \multicolumn{5}{|l|}{ Sex } \\
\hline Male/female & $0.768(0.431-1.370)$ & 0.372 & & \\
\hline \multicolumn{5}{|l|}{ Alcohol } \\
\hline Yes/no & $0.405(0.143-1.148)$ & 0.089 & & NS \\
\hline \multicolumn{5}{|l|}{ HBsAg } \\
\hline Positive/negative & $0.563(0.324-0.977)$ & 0.041 & & NS \\
\hline \multicolumn{5}{|l|}{ Anti-HCV } \\
\hline Positive/negative & $1.219(0.708-2.099)$ & 0.475 & & \\
\hline \multicolumn{5}{|l|}{$\mathrm{AFP}, \mathrm{ng} / \mathrm{mL}$} \\
\hline$>200 / \leq 200$ & $0.430(0.229-0.808)$ & 0.009 & $0.432(0.217-0.860)$ & 0.017 \\
\hline \multicolumn{5}{|l|}{$\mathrm{ALT}, \mathrm{U} / \mathrm{L}$} \\
\hline$>40 / \leq 40$ & $0.557(0.334-0.930)$ & 0.025 & & NS \\
\hline \multicolumn{5}{|l|}{ AST, U/L } \\
\hline$>40 / \leq 40$ & $0.524(0.313-0.877)$ & 0.014 & & NS \\
\hline \multicolumn{5}{|l|}{ Creatinine, $\mathrm{mg} / \mathrm{dL}$} \\
\hline$>2 / \leq 2$ & $0.301(0.040-2.262)$ & 0.243 & & \\
\hline \multicolumn{5}{|l|}{ Platelet, $10^{9} / \mathrm{L}$} \\
\hline$>150 / \leq 150$ & $0.717(0.424-1.211)$ & 0.213 & & NS \\
\hline \multicolumn{5}{|l|}{ ECOG status } \\
\hline$\geq 1 / 0$ & $1.248(0.359-4.338)$ & 0.728 & & \\
\hline \multicolumn{5}{|l|}{ ALBI grade } \\
\hline $2-3 / 1$ & $0.692(0.409-1.171)$ & 0.170 & & \\
\hline \multicolumn{5}{|l|}{ Use of CBCT } \\
\hline Yes/no & $0.983(0.572-1.690)$ & 0.952 & & \\
\hline \multicolumn{5}{|l|}{ 5-7 criteria } \\
\hline In/out & $2.529(1.222-5.237)$ & 0.012 & & NS \\
\hline \multicolumn{5}{|l|}{7 lesions criteria } \\
\hline In/out & $2.128(0.989-4.581)$ & 0.054 & & NS \\
\hline \multicolumn{5}{|l|}{ 7-11 criteria } \\
\hline High & 1 & & 1 & \\
\hline Intermediate & $5.299(1.987-14.134)$ & 0.001 & $4.617(1.769-12.760)$ & 0.002 \\
\hline Low & $10.381(3.979-27.082)$ & $<0.001$ & $8.675(3.294-22.844)$ & $<0.001$ \\
\hline
\end{tabular}

CBCT, cone-beam computed tomography; OR, odds ratio; CI, confidence interval; NS, not significant; TACE, transarterial chemoembolization; HBsAg, hepatitis B surface antigen; ALT, alanine aminotransferase; AST, aspartate aminotransferase; ECOG, Eastern Cooperative Oncology Group.

den developed tumor recurrence within 6 months after initial CR by first TACE session. The median OS of patients with low, intermediate, and high tumor burden were $74.6,35.5$, and 32.3 months, respectively, after achieving initial CR $(p=0.022)$.

\section{Comparison of Different Criteria of Tumor Burden in Predicting OS}

Table 3 demonstrates the discriminative value of different criteria of tumor burden in predicting OS. Among them, 7-11 criteria had the lowest AICc, highest homo- geneity value, and highest AUROC in predicting 1-, 2-, and 3-year mortality.

\section{Factors Associated with OS}

During the follow-up period, 489 of 632 patients (77.4\%) died. The overall median OS was 20.9 months (95\% confidence interval 18.0-24.7 months). In univariate analysis, age, AFP, AST levels, ECOG status, ALBI grade, 5-7 criteria, 7-11 criteria, and radiologic response were significantly associated with OS. In multivariate analysis, age $>60$ years $(\mathrm{HR}=1.366, p=0.006)$, AFP $>200$ 
Table 3. Comparison of various models of high tumor burden in predicting OS for intermediate-stage HCC patients undergoing TACE

\begin{tabular}{|c|c|c|c|c|c|}
\hline Model & $\begin{array}{l}\text { 1-year mortality } \\
\text { AUROC (95\% CI) }\end{array}$ & $\begin{array}{l}\text { 2-year mortality } \\
\text { AUROC (95\% CI) }\end{array}$ & $\begin{array}{l}\text { 3-year mortality } \\
\text { AUROC (95\% CI) }\end{array}$ & $\mathrm{AICc}$ & $\begin{array}{l}\text { Homo } \\
\chi^{2}\end{array}$ \\
\hline 7-11 criteria & $0.635(0.587-0.684)$ & $0.636(0.588-0.684)$ & $0.603(0.551-0.655)$ & 5499.859 & 35.870 \\
\hline Up-to-11 criteria & $0.621(0.571-0.671)$ & $0.613(0.564-0.663)$ & $0.597(0.545-0.650)$ & 5500.887 & 32.842 \\
\hline Up-to-7 criteria & $0.573(0.524-0.622)$ & $0.584(0.535-0.633)$ & $0.552(0.497-0.607)$ & 5516.390 & 17.339 \\
\hline 5-7 criteria & $0.550(0.499-0.601)$ & $0.540(0.489-0.590)$ & $0.523(0.469-0.577)$ & 5527.942 & 5.787 \\
\hline 7 lesions criteria & $0.534(0.483-0.585)$ & $0.530(0.479-0.580)$ & $0.517(0.463-0.571)$ & 5530.252 & 3.477 \\
\hline
\end{tabular}

AICc, corrected Akaike information criteria; HCC, hepatocellular carcinoma; TACE, transarterial chemoembolization; OS, overall survival; AUROC, area under the receiver operating characteristic curve.

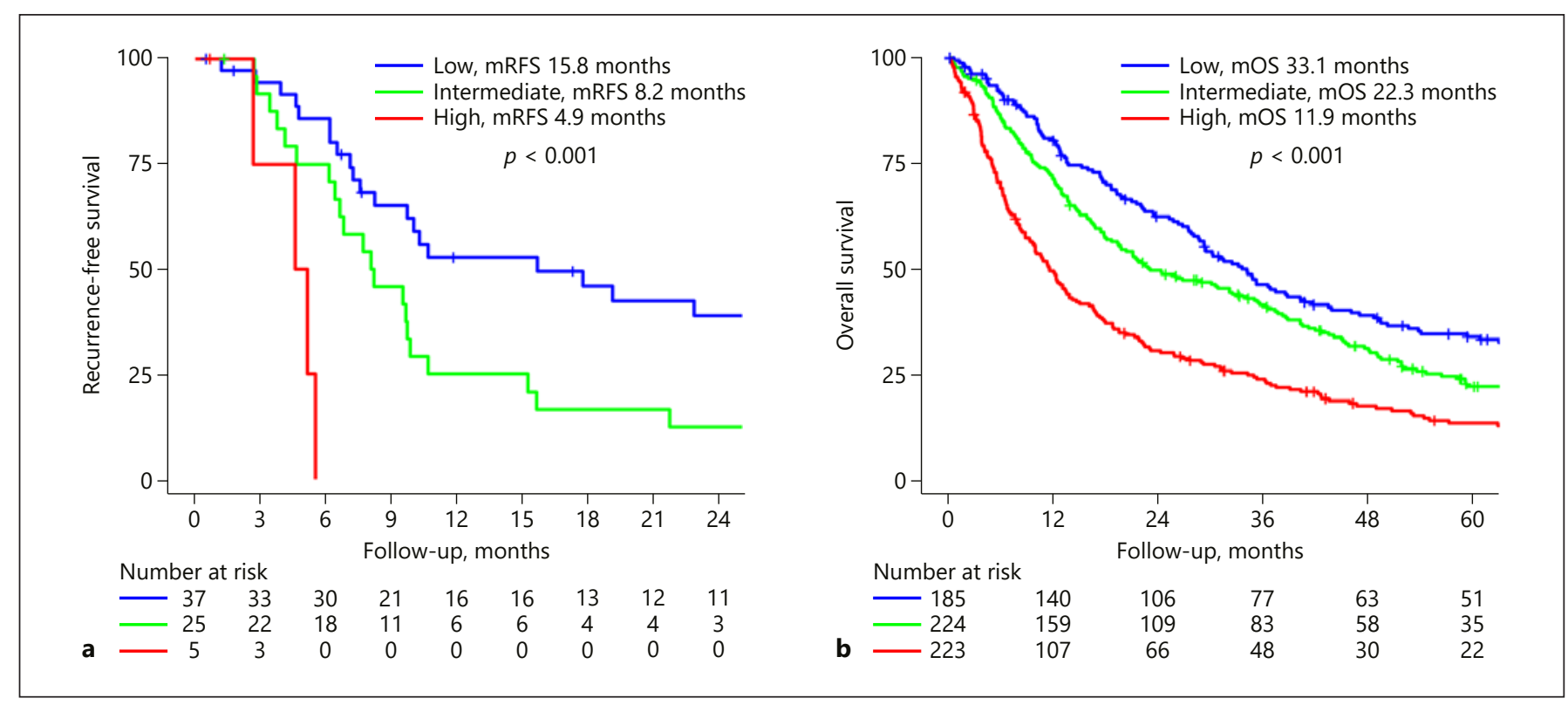

Fig. 2. Kaplan-Meier curves of RFS after complete response and OS of HCC patients undergoing TACE. a RFS stratified by 7-11 criteria. b OS stratified by 7-11 criteria. HCC, hepatocellular carcinoma; TACE, transarterial chemoembolization; OS, overall survival; RFS, recurrence-free survival.

$\mathrm{ng} / \mathrm{mL}(\mathrm{HR}=1.541, p<0.001)$, ECOG status $\geq 1(\mathrm{HR}=$ $1.778, p=0.015)$, ALBI grade $2-3(\mathrm{HR}=1.539, p<0.001)$, $7-11$ criteria (intermediate vs. high tumor burden, $\mathrm{HR}=$ $0.650, p<0.001$; low vs. high tumor burden, $\mathrm{HR}=0.520$, $p<0.001)$, and PD by mRECIST (HR $=1.787, p=0.001)$ were independent predictors of OS (Table 4). The median survival in patients with low, intermediate, high tumor burden classified by $7-11$ criteria were 33.1, 22.3, and 11.9 months, respectively ( $p<0.001$, Fig. $2 \mathrm{~b}$ ). The 3 -year survival rate in patients with low, intermediate, high tumor burden classified by $7-11$ criteria was $45.8,30.7$, and $23.4 \%$, respectively.

Redefining Tumor Burden of Intermediate HCC

\section{Liver Function Deterioration and Additional Treatment after Initial TACE}

Among the 502 patients with initial Child-Pugh class A, 91 (18.1\%) of them had liver function migration to Child-Pugh class B or C after repeated TACE procedures. The rate of liver function deterioration in patients with low, intermediate, and high tumor burden by $7-11$ criteria were $14.9,14.4$, and $24.4 \%$, respectively ( $p=0.021$, online suppl. Table 5).

After initial TACE, 91 (14.4\%) patients received subsequent curative treatment after successful down-staging by initial TACE, included liver transplantation $(n=13)$, 
Table 4. Univariate and multivariate analyses of factors associated with OS

\begin{tabular}{|c|c|c|c|c|}
\hline & \multicolumn{2}{|l|}{ Univariate } & \multicolumn{2}{|l|}{ Multivariate } \\
\hline & HR (95\% CI of HR) & $p$ value & HR (95\% CI of HR) & $p$ value \\
\hline \multicolumn{5}{|l|}{ Age, years } \\
\hline$>60 / \leq 60$ & $1.256(1.018-1.549)$ & 0.034 & $1.366(1.095-1.704)$ & 0.006 \\
\hline \multicolumn{5}{|l|}{ Sex } \\
\hline Male/female & $1.022(0.825-1.267)$ & 0.842 & & \\
\hline \multicolumn{5}{|l|}{ Alcohol } \\
\hline Yes/no & $1.170(0.908-1.509)$ & 0.224 & & \\
\hline \multicolumn{5}{|l|}{ HBsAg } \\
\hline Positive/negative & $1.003(0.838-1.200)$ & 0.974 & & \\
\hline \multicolumn{5}{|l|}{ Anti-HCV } \\
\hline Positive/negative & $0.988(0.819-1.192)$ & 0.900 & & \\
\hline \multicolumn{5}{|l|}{$\mathrm{AFP}, \mathrm{ng} / \mathrm{mL}$} \\
\hline$>200 / \leq 200$ & $1.724(1.433-2.074)$ & $<0.001$ & $1.541(1.268-1.874)$ & $<0.001$ \\
\hline \multicolumn{5}{|l|}{$\mathrm{ALT}, \mathrm{U} / \mathrm{L}$} \\
\hline$>40 / \leq 40$ & $1.054(0.880-1.263)$ & 0.569 & & \\
\hline \multicolumn{5}{|l|}{$\mathrm{AST}, \mathrm{U} / \mathrm{L}$} \\
\hline$>40 / \leq 40$ & $1.370(1.127-1.666)$ & 0.002 & & NS \\
\hline \multicolumn{5}{|l|}{ Creatinine, $\mathrm{mg} / \mathrm{dL}$} \\
\hline$>2 / \leq 2$ & $1.282(0.843-1.952)$ & 0.246 & & \\
\hline \multicolumn{5}{|l|}{ Platelet, $10^{9} / \mathrm{L}$} \\
\hline$>150 / \leq 150$ & $1.020(0.852-1.220)$ & 0.831 & & \\
\hline \multicolumn{5}{|l|}{ ECOG status } \\
\hline$\geq 1 / 0$ & $1.665(1.094-2.533)$ & 0.017 & $1.778(1.117-2.829)$ & 0.015 \\
\hline \multicolumn{5}{|l|}{ ALBI grade } \\
\hline $2-3 / 1$ & $1.592(1.301-1.948)$ & $<0.001$ & $1.539(1.249-1.897)$ & $<0.001$ \\
\hline \multicolumn{5}{|l|}{ Use of CBCT } \\
\hline Yes/no & $0.939(0.770-1.146)$ & 0.537 & & \\
\hline \multicolumn{5}{|l|}{$5-7$ criteria } \\
\hline In/out & $0.778(0.637-0.951)$ & 0.014 & & NS \\
\hline \multicolumn{5}{|l|}{7 lesions criteria } \\
\hline In/out & $0.809(0.651-1.006)$ & 0.057 & & NS \\
\hline \multicolumn{5}{|l|}{ 7-11 criteria } \\
\hline High & 1 & & 1 & \\
\hline Intermediate & $0.632(0.513-0.779)$ & $<0.001$ & $0.650(0.520-0.813)$ & $<0.001$ \\
\hline Low & $0.516(0.413-0.646)$ & $<0.001$ & $0.520(0.407-0.662)$ & $<0.001$ \\
\hline \multicolumn{5}{|c|}{ Radiologic response to initial TACE } \\
\hline Complete response & 1 & & 1 & \\
\hline PR & $1.187(0.839-1.678)$ & 0.333 & $0.927(0.652-1.319)$ & 0.674 \\
\hline SD & $1.697(1.205-2.390)$ & 0.002 & $1.217(0.852-1.738)$ & 0.279 \\
\hline $\mathrm{PD}$ & $2.243(1.601-3.143)$ & $<0.001$ & $1.787(1.266-2.522)$ & 0.001 \\
\hline
\end{tabular}

liver resection $(n=40)$, and radiofrequency ablation $(n=$ 38 ), while 144 patients (22.8\%) patients received subsequent systemic therapies after TACE failure or PD. The rates of transition to curative treatment in patients with low, intermediate, and high tumor burden by 7-11 crite- ria were $18.9,15.2$, and $9.9 \%$, respectively $(p=0.011)$. The corresponding rates of transition to systemic therapies were $21.1,22.3$, and $24.7 \%$, respectively ( $p=0.408$ online suppl. Table 5). 


\section{Discussion and Conclusion}

In this study, we compared the prognostic value of different criteria of tumor burden, in terms of predicting radiologic response and survival after TACE for patients with intermediate-stage HCC. We showed that significantly different proportion of patients were classified as high tumor burden by different criteria, which was $70 \%$ by up-to- $7,35.5 \%$ by up-to- $11,26.4 \%$ by $5-7$, and $21 \%$ by 7 lesions criteria. If patients with high tumor burden were defined as unsuitable for TACE, only about $30 \%$ of patients with intermediate-stage HCC could consider TACE as initial therapy by the up-to-7 criteria.

Several criteria of tumor burden have been proposed, such as 5-7, 7 lesions, up-to-7, and up-to-11 criteria. Yamakado et al. [25] also demonstrated that 4 tumors measuring $7 \mathrm{~cm}$ or smaller (4-of- $7 \mathrm{~cm}$ criterion) was a favorable prognostic factor for intermediate-stage HCC patients. In our study, the prognostic value of 5-7 and 7 lesions criteria in discriminating response and survival were much less significant than the up-to-7 or up-to-11 criteria (Fig. 1; Table 3). The up-to-7 criteria may select patients with the highest chance of $\mathrm{CR}$, while up-to-11 criteria may select patients with the lowest chance of $\mathrm{CR}$ and the highest chance of $\mathrm{PD}$. To further discriminate the chance of radiologic response, we combined up-to-7 and up-to-11 and proposed the 7-11 criteria, which stratified patients into low, intermediate, and high tumor burden. The 7-11 criteria could better stratify the chance of radiologic response, especially CR, among the 3 groups. By multivariate analysis, we showed that 7-11 criteria were the most predominant predictor of achieving $\mathrm{CR}$, objective response, and $\mathrm{PD}$ after the first session of TACE. Of note, we found that the ORR and DCR were similar between patients with intermediate and low tumor burden by 7-11 criteria, suggesting that patients beyond up-to-7 but within up-to- 11 criteria could still benefit from TACE in terms of getting radiologic response and disease control.

In patients with intermediate-stage HCC undergoing TACE, complete response could still be achieved in a small proportion of patients, even in patients with intermediate and high tumor burden. Patients achieving CR were shown to have a significantly better long-term survival $[16,17]$. In this study, $11.5 \%$ of patients could achieve CR by the first session of TACE, but $80.6 \%$ of them developed HCC recurrence during the follow-up period, suggesting that stringent follow-up remains needed in patients achieving CR after TACE. Our data showed that the 7-11 criteria could significantly stratify the

Redefining Tumor Burden of Intermediate HCC chance of CR, the risk of recurrence after $\mathrm{CR}$, and the OS in subgroup patients achieving CR.

We further compared the prognostic value of different criteria of tumor burden in OS after TACE. We showed that up-to- 11 criteria were better than up-to-7 criteria in predicting OS, while the new 7-11 criteria provided the best prognostic value, which had the lowest AICc, highest homogeneity, and highest AUROC in predicting 1-, 2-, and 3 -year mortality. These data suggested that 7-11 criteria were the best to discriminate OS among the 5 models. By multivariate analysis, the 7-11 criteria could significantly predict OS, independent of age, AFP levels, liver function reserve and radiologic response. The OS was equally distributed in patients with low, intermediate, and high tumor burden, with median OS was 33.1, 22.3, and 11.9 months, respectively (Fig. 2b). Although the difference of AIC between 7 and 11 and up-to 11 criteria was small, the 7-11 criteria had higher AUROC to predict 1-, 2-, and 3-year mortality and higher Homo $\chi^{2}$ than other criteria of tumor burden. Besides, the 7-11 criteria not only had the best predictive role in overall survival, but also presented the best discriminative value to predict radiologic response. Our data suggest that 7-11 criteria was superior to up-to-7 and up-to-11 criteria and could help to improve treatment decision making for intermediate-stage HCC.

A recent multicenter study showed that the CR rate ranged from 1.5 to $53.4 \%$ among 8 multicenter cohorts [17]. There were few studies reporting the CR and OS rates in patients within up-to-7. Kim et al. [16] reported a median OS of 43.6 months and a CR rate of $59.2 \%$ in patients with tumor burden beyond Milan criteria but in up-to-7 criteria. However, in this study, the overall CR rate was $52.5 \%$, which was much higher than the average CR rate reported previously. Besides, the study by Kim et al. [16] also revealed that tumor burden by Milan criteria and up-to-7 criteria significantly affected the CR rate and survival of patients receiving TACE. The heterogeneity of patient population and different TACE techniques might contribute to the wide range of CR rates and OS among different studies $[25,26]$. In our study, the CR and OS rates were comparable to previous reports, and we could significantly discriminate $\mathrm{CR}$ and overall survival rates by $7-11$ criteria.

Liver function deterioration after repeated TACE is an important concern in patients with intermediatestage HCC. In this study, we observed $18.1 \%$ of patients had liver function migration from Child-Pugh class A to Child-Pugh class B or C after repeated TACE procedures, and patients with higher tumor burden had a significantly higher risk of liver function deterioration. Additional treatment after initial TACE may have strong 
influence on OS. In this study, 91 (14.4\%) patients had successful down-staging by initial TACE and could receive subsequent curative treatment, and tumor burden by 7-11 criteria may also stratify the possibility to receive curative treatment after successful down-staging by initial TACE, which may lead to a better overall survival. On the other hand, 144 patients $(22.8 \%)$ patients received subsequent systemic therapies after TACE failure or PD. We observed comparable percentage of patients with low, intermediate and high tumor burden by 7-11 criteria received subsequent systemic therapies, indicating that the impact of systemic therapies would not change the prognostic value of tumor burden by 7-11 criteria.

Recent advances in systemic therapies have changed the paradigm for the treatment of intermediate-stage HCC. Early introduction of systemic therapy should be considered for patients unsuitable or refractory to TACE treatment $[7,9,27]$. It is generally accepted that high tumor burden indicates TACE-unsuitable, but the definition of high tumor burden was not well-defined in intermediate-stage HCC. The recent Asian-Pacific consensus adopted up-to-7 criteria to describe tumor burden and guide the decision of treatment selection [8]. However, up-to-7 criteria were originally developed for liver transplantation [13], which was usually applied in patients with early-stage HCC. In this study, we showed that the majority of patients in intermediate-stage HCC were actually beyond up-to-7 criteria. Besides, up-to-7 criteria did not show the best discriminative value in predicting response and survival after TACE. Therefore, high tumor burden in intermediate-stage HCC should be redefined. Our data showed that patients within up-to-7 had the best radiologic response and OS, but a significant proportion of patients with intermediate tumor burden by $7-11$ criteria may still get benefit from TACE. In contrast, patients beyond up-to-11 indeed had worse outcomes. With near $50 \%$ of ORR in patients with intermediate tumor burden, this subgroup should not be considered as TACE-unsuitable by using conventional up-to-7 criteria.

Currently, several strategies of early introducing systemic therapies in intermediate HCC have been proposed, such as the combination of TACE with targeted therapy [18] or initial targeted therapy followed by subsequent TACE [28]. For patients with intermediate tumor burden, our data suggest that TACE could still be considered in this population, but the $\mathrm{CR}$ rate would be compromised. Adjuvant systemic treatment might be considered to enhance tumor control. For patients with high tumor burden, systemic therapies could be considered as the initial treatment due to TACE-unsuitable, subsequent TACE might be considered if downsizing the tumor burden can be achieved.

There are some limitations in this study. First, this is a retrospective study from a single medical center in Taiwan. Our results require prospective external validation in the future. Second, the subsequent treatments after first session TACE were not analyzed in this study, which might have a significant impact on OS. Especially, newer systemic therapies have been introduced in recent years, which might further prolong patients' survival after progression to advanced stage. Third, the impact of TACE on liver function deterioration among patients with different tumor burden was not evaluated. Although TACE could still provide radiologic responses in a certain proportion of patients with intermediate and high tumor burden, the objective responses and preserving liver function are equally important to prolong OS [5]. The balance between safety and efficacy of TACE for HCC patients with intermediate and high tumor burden warrants further attention. Forth, although early initiation of systemic therapies could be considered in patients with intermediate or high tumor burden, due to the National Health Insurance policy in Taiwan, early initiation of systemic therapies for patients with intermediate or high tumor burden were not reimbursed. Therefore, we could not evaluate the efficacy of early initiation of systemic therapies in our patients.

In conclusion, conventional definitions of high tumor burden are not optimal for patients with intermediatestage HCC. We propose new 7-11 criteria, which were shown to be most discriminative in predicting radiologic response and $O S$ in patients with intermediate-stage HCC undergoing TACE. The treatment strategy in intermediate-stage HCC could be reconsidered based on the new 7-11 criteria.

\section{Acknowledgement}

The authors thank the Clinical Research Core Laboratory, Taipei Veterans General Hospital, for providing their facilities to conduct this study.

\section{Statement of Ethics}

This study gained consent to the Institutional Review Board of Taipei Veterans General Hospital (IRB number: 2019-10-001BC) and was conducted ethically in accordance with the World Medi- 
cal Association Declaration of Helsinki. Because of the retrospective nature of this study, the Institutional Review Board waived the need for written informed consent.

\section{Conflict of Interest Statement}

Y.-H.H. has received research grants from Gilead Sciences and Bristol-Meyers Squibb and honoraria from Abbvie, Gilead Sciences, Bristol-Meyers Squibb, Ono Pharmaceutical, Merck Sharp \& Dohme, Eisai, Eli Lilly, Ipsen, and Roche and has served in an advisory role for Abbvie, Gilead Sciences, Bristol-Meyers Squibb, Ono Pharmaceuticals, Eisai, Eli Lilly, Ipsen, Merck Sharp \& Dohme, and Roche. Other authors declare no conflicts of interest.

\section{Funding Sources}

The study was supported by grants from Taipei Veterans General Hospital, Taipei, Taiwan (V109C-056, V110C-094), and Ministry of Science and Technology, Taiwan (MOST 109-2628-B-075022, MOST 109-2314-B-010-034-MY3).

\section{Author Contributions}

Y.-W. Hung: data acquisition, analysis and interpretation of data, drafting of the manuscript, and statistical analysis. C.-T. Chi, R.-C. Lee, C.-A. Liu, N.-C. Chiu, H.-N. Hwang, Y. Chao, and M.-C. Hou: data acquisition. I.-C. Lee and Y.-H. Huang: study concept and design, obtained funding, critical revision of the manuscript for important intellectual content, and study supervision.

\section{References}

1 Arnold M, Abnet CC, Neale RE, Vignat J, Giovannucci EL, McGlynn KA, et al. Global burden of 5 major types of gastrointestinal cancer. Gastroenterology. 2020;159(1):33549.e15.

2 European Association for the Study of the Liver. EASL clinical practice guidelines: management of hepatocellular carcinoma. J Hepatol. 2018;69(1):182-236.

3 Heimbach JK, Kulik LM, Finn RS, Sirlin CB, Abecassis MM, Roberts LR, et al. AASLD guidelines for the treatment of hepatocellular carcinoma. Hepatology. 2018;67(1):358-80.

4 Omata M, Cheng AL, Kokudo N, Kudo M, Lee JM, Jia J, et al. Asia-Pacific clinical practice guidelines on the management of hepatocellular carcinoma: a 2017 update. Hepatol Int. 2017;11(4):317-70.

5 Piscaglia F, Ogasawara S. Patient selection for transarterial chemoembolization in hepatocellular carcinoma: importance of benefit/ risk assessment. Liver Cancer. 2018;7(1):10419.

6 Koroki K, Ogasawara S, Ooka Y, Kanzaki H, Kanayama K, Maruta S, et al. Analyses of intermediate-stage hepatocellular carcinoma patients receiving transarterial chemoembolization prior to designing clinical trials. Liver Cancer. 2020;9(5):596-612.

7 Kudo M, Ueshima K, Chan S, Minami T, Chishina $\mathrm{H}$, Aoki T, et al. Lenvatinib as an initial treatment in patients with intermediate-stage hepatocellular carcinoma beyond up-to-seven criteria and child-pugh a liver function: a proof-of-concept study. Cancers. 2019;11(8): 1084.

8 Kudo M, Han KH, Ye SL, Zhou J, Huang YH, Lin SM, et al. A Changing paradigm for the treatment of intermediate-stage hepatocellular carcinoma: Asia-Pacific primary liver cancer expert consensus statements. Liver Cancer. 2020;9(3):245-60.
9 Galle PR, Tovoli F, Foerster F, Wörns MA, Cucchetti A, Bolondi L. The treatment of intermediate stage tumours beyond TACE: from surgery to systemic therapy. J Hepatol. 2017;67(1):173-83.

10 Bolondi L, Burroughs A, Dufour JF, Galle PR, Mazzaferro V, Piscaglia F, et al. Heterogeneity of patients with intermediate (BCLC B) Hepatocellular Carcinoma: proposal for a subclassification to facilitate treatment decisions. Semin Liver Dis. 2012;32(4):348-59.

11 Kudo M, Arizumi T, Ueshima K, Sakurai T, Kitano M, Nishida N. Subclassification of BCLC B stage hepatocellular carcinoma and treatment strategies: proposal of modified Bolondi's subclassification (Kinki criteria). Dig Dis. 2015;33(6):751-8.

12 Hucke F, Pinter M, Graziadei I, Bota S, Vogel W, Müller C, et al. How to STATE suitability and START transarterial chemoembolization in patients with intermediate stage hepatocellular carcinoma. J Hepatol. 2014;61(6):128796.

13 Mazzaferro V, Llovet JM, Miceli R, Bhoori S, Schiavo M, Mariani L, et al. Predicting survival after liver transplantation in patients with hepatocellular carcinoma beyond the Milan criteria: a retrospective, exploratory analysis. Lancet Oncol. 2009;10(1):35-43.

14 Lee IC, Hung YW, Liu CA, Lee RC, Su CW, Huo TI, et al. A new ALBI-based model to predict survival after transarterial chemoembolization for BCLC stage B hepatocellular carcinoma. Liver Int. 2019;39(9):1704-12.

15 Kim JH, Shim JH, Lee HC, Sung KB, Ko HK, Ko GY, et al. New intermediate-stage subclassification for patients with hepatocellular carcinoma treated with transarterial chemoembolization. Liver Int. 2017;37(12):1861-8.
16 Kim BK, Kim SU, Kim KA, Chung YE, Kim MJ, Park MS, et al. Complete response at first chemoembolization is still the most robust predictor for favorable outcome in hepatocellular carcinoma. J Hepatol. 2015;62(6):1304-10.

17 Han G, Berhane S, Toyoda H, Bettinger D, Elshaarawy O, Chan AWH, et al. Prediction of survival among patients receiving transarterial chemoembolization for hepatocellular carcinoma: a response-based approach. Hepatology. 2020;72(1):198-212.

18 Kudo M, Ueshima K, Ikeda M, Torimura T, Tanabe N, Aikata H, et al. Randomised, multicentre prospective trial of transarterial chemoembolisation (TACE) plus sorafenib as compared with TACE alone in patients with hepatocellular carcinoma: TACTICS trial. Gut. 2020;69(8):1492-501.

19 Bruix J, Sherman M. Management of hepatocellular carcinoma: an update. Hepatology. 2011;53(3):1020-2.

20 Lee IC, Huo TI, Huang YH, Chao Y, Li CP, Lee PC, et al. Transarterial chemoembolization can prolong survival for patients with metastatic hepatocellular carcinoma: a propensity score matching analysis. Hepatol Int. 2012;6(4):753-62.

21 Tsai YC, Shih JH, Hwang HE, Chiu NC, Lee RC, Tseng HS, et al. Early prediction of 1-year tumor response of hepatocellular carcinoma with lipiodol deposition pattern through post-embolization cone-beam computed tomography during conventional transarterial chemoembolization. Eur Radiol. 2021.

22 Johnson PJ, Berhane S, Kagebayashi C, Satomura S, Teng M, Reeves HL, et al. Assessment of liver function in patients with hepatocellular carcinoma: a new evidence-based approach-the ALBI grade. J Clin Oncol. 2015;33(6):550-8.

23 Lencioni R, Llovet JM. Modified RECIST (mRECIST) assessment for hepatocellular carcinoma. Semin Liver Dis. 2010;30(1):5260. 
24 Hand DJ, Till RJ. A simple generalisation of the area under the ROC curve for multiple class classification problems. Mach Learn. 2001;45(2):171-86.

25 Yamakado K, Miyayama S, Hirota S, Mizunuma K, Nakamura K, Inaba Y, et al. Subgrouping of intermediate-stage (BCLC stage B) hepatocellular carcinoma based on tumor number and size and Child-Pugh grade correlated with prognosis after transarterial chemoembolization. Jpn J Radiol. 2014;32(5):260-5.
26 Saito N, Tanaka T, Nishiohuku H, Sato T, Masada T, Matsumoto T, et al. Transarterialchemoembolization remains an effective therapy for intermediate-stage hepatocellular carcinoma with preserved liver function. Hepatol Res. 2020;50(10):1176-85.
27 Arizumi T, Ueshima K, Minami T, Kono M, Chishina $\mathrm{H}$, Takita $\mathrm{M}$, et al. Effectiveness of sorafenib in patients with transcatheter arterial chemoembolization (TACE) refractory and intermediate-stage hepatocellular carcinoma. Liver Cancer. 2015;4(4):253-62.

28 Kudo M. A new treatment option for intermediate-stage hepatocellular carcinoma with high tumor burden: initial lenvatinib therapy with subsequent selective TACE. Liver Cancer. 2019;8(5):299-311. 